\title{
ON OPERATION OF SELF-PHASED ARRAYS UNDER CONDITIONS OF MULTIPATH PROPAGATION
}

\author{
S.L. Loyka*
}

\begin{abstract}
This paper gives an analysis of operation of a self-phased array under conditions of multipath propagation (either reflections or jamming signals). A general method for the calculation of directivity of such an array has been proposed. Influence of reflections by way of the pilot signal channel as well as by way of the primary signal channel is taken into account. Some limitations of the approach proposed before have been pointed out and overcome in this paper. It has been shown that an essential reduction in directivity is possible in the case of weak pilot signal or large (close to unity) reflection coefficient.
\end{abstract}

\section{Introduction}

Self-phased arrays have lately received wide application in telecommunications systems [1]. They have a number of advantages in comparison with conventional antenna arrays. However, the electromagnetic environment can essentially influence their operation. Presence of reflections (multipath propagation) or jamming (interference) signals at the pilot signal frequency can lead to significant deterioration of a self-phased array operation. Both the pilot signal channel and the primary (information) signal channel can substantially influence on the self-phased array operation.

Directivity of self-phased arrays under conditions of multipath propagation have been investigated in [1]. However, the influence of reflections by way of the pilot signal channel has not been taken into account and the expressions for directivity of such an array have been obtained under assumptions which are not correct in some cases [2]. The purpose of the present paper is to develop more rigorous and general method of directivity calculation and to establish limits of the method given in [1].

We will consider these issues in more detail using the receiving self-phased array structure given in [1] (see Figure 1). In this structure, each self-phasing unit consists of two filters tuned to the frequency of the primary and pilot signals, a limiter and a mixer (see Figure 2). The primary and pilot signals are passed to the mixer input. A signal at intermediate frequency which is equal to the difference of primary and pilot signal frequencies is present at the mixer output.

In the case of the threshold amplitude characteristic of the pilot-signal channel, directivity of self-phased array under conditions of multipath propagation can be written as follows $[1,3]$ :

$$
G=G_{p} \cdot G_{s} \cdot G_{0}
$$

where $G_{0}$ - is the array directivity in the absence of reflections $\left(G_{0} \approx N\right.$ when directivity of an array element is close to unity, further we will consider just this specific case; $N$ - is the number

Senior Scientist, Ph.D., Electromagnetic Compatibility Laboratory, Belorussian State University of Informatics and Radioelectronics, P.Brovki Str. 6, 220027, Minsk, Republic of Belarus, e-mail: loyka@nemc.belpak.minsk.by 
of array elements); $G_{p}$ - is reduction in directivity due to cutting off some channels (influence of reflections

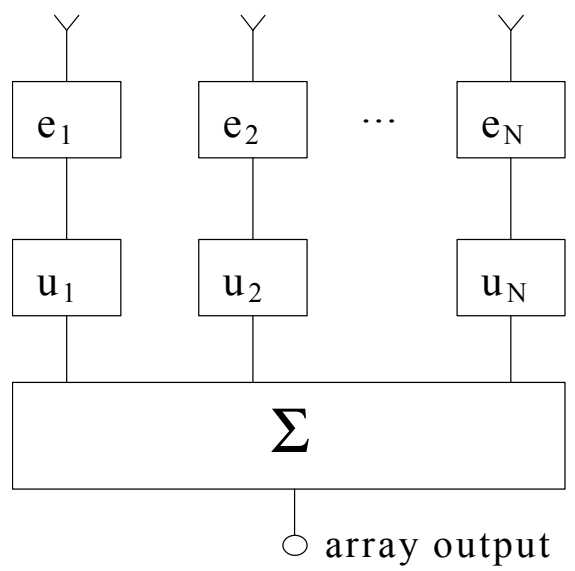

Figure 1. Self-phased array structure. $\mathrm{e}_{1}$ - $\mathrm{e}_{\mathrm{N}}$ - array elements; $\mathrm{u}_{1}-\mathrm{u}_{\mathrm{N}}$ - selfphasing units; $\Sigma$ - adder.

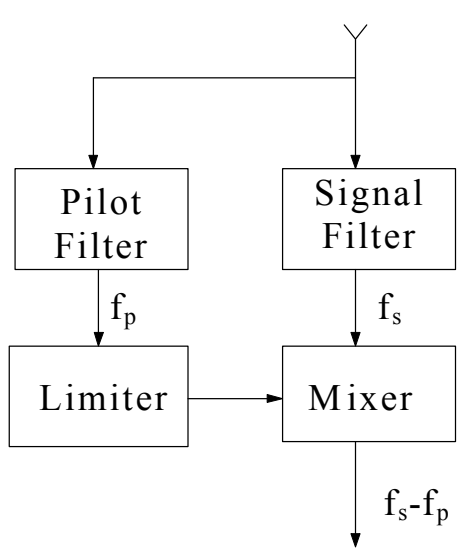

Figure 2. Self-phasing unit structure. $f_{s}$ , $\mathrm{f}_{\mathrm{p}}$ - primary signal and pilot frequencies consequently.

by way of the pilot signal channel, see section 3); $G_{s}$ - is reduction in directivity owing to variations in amplitude and phase of the primary signal (influence of reflections by way of the primary signal channel, see section 2).

\section{Influence Of The Primary Signal Channel On The Array Directivity}

The general expression for the reduction in the array directivity by way of the primary signal channel is as follows [2]:

$$
G_{s}=\frac{1}{N}\left|\sum_{n=1}^{N} \sqrt{\left(1+a^{2}+2 \cdot a \cdot \cos \left(\vartheta_{n s}\right)\right)} \cdot \exp \left(j\left(\psi_{n s}-\psi_{n p}\right)\right)\right|
$$

where $a=A_{r} / A_{d}$ - is the reflection factor (the ratio of the amplitudes of reflected $A_{r}$ and $\operatorname{direct} A_{d}$ signal), $\vartheta_{\text {ns }}$ - is the phase difference between the reflected and direct primary signal in n-th array element, $\psi_{n s}$ and $\psi_{n p}$ - are the phases of the total (direct plus reflected) primary and pilot signals accordingly in n-th array element. The phase difference between the reflected and direct primary signal $\vartheta_{n s}$ as well as the phases of the total primary and pilot signals $\left(\psi_{n s}\right.$ and $\left.\psi_{n p}\right)$ depend on change in the phase difference between neighbouring elements $\Delta \vartheta_{\mathrm{s}}=\vartheta_{\mathrm{n}+1, \mathrm{~s}}-\vartheta_{\mathrm{ns}}$ and on the phase difference in the first elements $\vartheta_{1 \mathrm{~s}}$ and on the array geometry (formulas for this quantities can be found in [2]). In its turn, $\vartheta_{1 \mathrm{~s}}$ and $\Delta \vartheta_{\mathrm{s}}$ depend on the direct and reflected signal arrival direction and the difference of direct and reflected path length. So, we can carry out averaging of equation (2) over $\vartheta_{1 \mathrm{~s}}$ and over $\Delta \vartheta_{\mathrm{s}}$ 


$$
G_{S}\left(a, \Delta \vartheta_{s}\right)=\frac{1}{2 \pi} \cdot \int_{0}^{2 \pi} G_{S}\left(a, \vartheta_{1 s}\right) d \vartheta_{1 s} \quad(3 \mathrm{a}) \quad, \quad G_{S}(a)=\frac{1}{2 \pi} \cdot \int_{0}^{2 \pi} G_{S}\left(a, \Delta \vartheta_{S}\right) d \Delta \vartheta_{S}
$$

Eq. (3b) is valid for the linear array with uniformly-located elements. The averaging over the arrival direction angle(s) should be made for the array with more complicated geometry. The averaging over $\vartheta_{1 \mathrm{~s}}$ is necessary because of random character of the reflection point position and the path length difference. The averaging over $\Delta \vartheta_{\mathrm{s}}$ is necessary because of random character of the arrival directions of the direct and reflected signals. If we know the arrival directions of the direct and reflected signals, then Eq.(3a) should be used. Otherwise we should use Eq.(3b).

Using equations (1)-(3) and some assumptions which simplify the problem, it is possible to derive equations for the array directivity given in [1]. Comparison of the present method with that given in [1] shows that there are 5 reasons limiting the application of the latter method: (1) variation from element to element in the change of the primary signal amplitude because of reflection has not been taken into account, (2) variation from element to element in the difference of the phases of the total and direct signals has not been taken into account, (3) the amplitude of the total primary signal at the array output is not equal to the sum of the amplitudes of the direct and reflected primary signals because of the presence of phase factors, (4) the assumption about uncorrelation of the variation from element to element in the difference of the phases of the total and direct signals is not true in many cases (for instance, in the case of the linear array with uniformly-located elements these variations are identical for all elements), (5) the method given in [1] is inapplicable if variation from element to element in the difference of the phases of the total and direct pilot signals $\Delta \vartheta_{\mathrm{p}}$ are small enough (namely, when $N \cdot \Delta \vartheta_{p}<2 \pi$ ).

\section{Influence Of The Pilot Signal Channel On The Array Directivity}

Since the pilot signal is subjected to a limiting process prior to the mixer, we can assume that the channel of the pilot signal has threshold performance: if the amplitude of the pilot signal at the input of a self-phasing unit exceeds the threshold value (the receive sensitivity) $A_{\min }$ then the channel operates properly (provided that the amplitude of the primary signal exceeds the sensitivity level); if the amplitude of the pilot signal is lower than the threshold level then the channel is cut off (the "drop out" occurs). In fact, $A_{\min }$ is the cut-off threshold of the channel at the input of the self-phasing unit. Cutting off some channels will reduce the level of the primary signal at the array output and, consequently, decrease the directivity.

Decrease in the array directivity due to cutting off some channels is described by the parameter $G_{p}$ which can be defined as:

$$
G_{p}=1-\frac{N_{d}}{N}
$$


where $N_{d}$ - is the number of the cut-off channels. This equation can be justified as follows (now we do not take into account $\left.G_{s}\right)$. If there are $N_{d}$ cut-off channels, then $G=N-N_{d}=N\left(1-N_{d} / N\right)=$ $G_{0} G_{p}$, where $G_{p}$ is given by (4).

Let us now consider one channel of the array. The condition for a proper channel operation looks like:

$$
J=\frac{A_{\text {total }}}{A_{\min }}=\beta \cdot \sqrt{1+a^{2}+2 \cdot a \cdot \cos \theta} \geq 1
$$

where $A_{\text {total }}$ - is the amplitude of the total pilot signal at the input of the self-phasing unit; $\beta=A / A_{\text {min }}$ - is a relative level of the direct pilot signal; $\grave{A}$ - is amplitude of the direct pilot signal; $\theta$ - is phase shift between the direct and reflected pilot signal at the input of the array element. Here we assume that array elements are omnidirectional (the directional properties of elements can be easily taken into account if necessary) and that all channels have identical parameters. The condition for cutting off the channel is $J<1$.

Analysis of the last expression and (5) reveals a peculiar "double threshold effect". The essence of this effect is explained in the following. If $\beta \geq 1 /(1-a)$, then all channels will operate properly and $G_{p}=1$. In this case the presence of reflection does not result in the deterioration of array performance owing to the pilot signal influence. If $\beta<1 /(1+a)$, then all channels of the array will be cut off and $G_{p}=0$. If $1 /(1+a) \leq \beta \leq 1 /(1-a)$, then $0 \leq G_{p} \leq 1$. The specific magnitude of $G_{p}$ is determined by array geometry and the value of $\theta$ for each channel.

Using Eq.(4), (5), we can make the deterministic evaluation of $\mathrm{G}_{\mathrm{p}}$ for given $a, \beta$ and $\theta$ (the last quantity is specific for each element of the array and can be calculated with the use of wellknow geometric formulas; see, for instance, [2]). When the path difference between the direct and reflected signals or the phase shift during reflection is random, the reduction in directivity is random too. Let us calculate the average reduction in directivity using the following method. First, the probability of cutting off one channel is calculated. Then we calculate the average number of cut-off channels and the average reduction in directivity (this method is valid when $\mathrm{N}$ $>10$ ). Assuming the uniform distribution of $\theta$ at the interval $[-\pi, \pi]$ (this assumption is valid for many practical cases, namely when the path difference between the direct and the reflected signal is comparable to or greater than the wavelength), we get the following equation for the average value of $G_{p}$

$$
\bar{G}_{p}=\frac{1}{\pi} \cdot \arccos \left(\frac{1-\beta^{2}\left(1+a^{2}\right)}{2 \cdot a \cdot \beta^{2}}\right)
$$

As analysis of this equation shows, the average reduction in directivity does not exceed $3 \mathrm{~dB}$ for $\beta \geq 1$. A significant reduction in directivity is possible for a low level pilot signal (a small value of $\beta$ ) and for a large reflection coefficient. If the level of the pilot signal is lower than the threshold level, presence of reflections leads to an increase of average directivity in comparison with the case without reflections when directivity is equal to zero. 
Equation (6) and the expressions describing the "double threshold effect" can be used for array design as follows. If there is a reflection and the reflection coefficient $a$ is known, then it is necessary to decrease the cut-off threshold (the receive sensitivity level) of the pilot signal channel in order to prevent cutting off the channels and, accordingly, reducing the directivity.

The sensitivity level is to be decreased to $A_{\min }^{*}=A_{\min } \cdot(1-a)$. In this case all units will operate properly and, accordingly, reduction in the directivity will not occur (provided that the array operates properly in the absence of reflection). If the permissible average reduction in directivity $\bar{G}_{p}$ and reflection coefficient $a$ are specified, then the required decrease (or increase) in sensitivity level can be calculated with the use of Equation (6):

$$
A_{\min }^{*}=A_{\min } \cdot \sqrt{1+a^{2}+2 \cdot a \cdot \cos \left(\pi \bar{G}_{p}\right)}
$$

\section{Conclusion}

The analysis of self-phased arrays under conditions of multipath propagation has shown that the pilot signal channel as well as primary signal channel can significantly influence the array directivity. It is worthwhile using the average reduction in the array directivity at the expense of cutting off some channels as a measure of the pilot channel influence. The greatest reduction in the array directivity is observed for the case of small amplitudes of the pilot signal or for a large reflection coefficient. If the amplitude of the pilot signal exceeds the sensitivity level, the average reduction in directivity does not exceed $3 \mathrm{~dB}$.

The obtained results can be used for analysis and design of self-phased arrays in complicated electromagnetic environment, e.g. in the presence of reflections (multipath propagation) or jamming signals at the frequency of the pilot signal.

It should be noted that there are additional lobes in a self-phased array because of the mixing process in self-phasing units, which should be taken into account during estimation of interference immunity of such an array. Main characteristics of such an additional lobes can be found in [4-5].

\section{References}

1. Brennan P.V. An Experimental and Theoretical Study of Self-Phased Arrays in Mobile Satellite Communications, IEEE Trans. on AP, v.37, pp. 1370-1376.

2. Loyka S.L. Directivity of Self-Phased Arrays in Conditions of Multipath Propagation, Proceedings of the Seventh International Conference on HF Radio Systems and Techniques, East Midlands Conference Centre, Nottingham, UK, 7-10 July, 1997.

3. Loyka S.L. Influence of Pilot Signal on Directivity of Self-Phased Arrays Under Conditions of Multipath Propagation, accepted for publication in IEEE EMC Transactions.

4. Loyka S.L. Characteristics of a receiving intermodulation channel of active array antennas, International Journal of Electronics, v.80, pp.595-602. 
5. Loyka S.L. Interference Immunity of Active Array Antennas in Rigid Electromagnetic Environment, Proceeding of the XI International Microwave Conference "MIKON-96", Poland, Warsaw, 1996, vol.1, pp.57-61 